\title{
Un marco teórico para delimitar el concepto de enoturismo sostenible desde un enfoque multi-nivel
}

\section{A theoretical framework to delimit the concept of sustainable wine tourism from a multi-level approach}

\author{
Nieves García-Casarejos ${ }^{1}$ Pilar Gargallo ${ }^{1}$ y Begoña Cabanes ${ }^{1}$ \\ ${ }^{1}$ Facultad de Economía y Empresa, 50008 Universidad de Zaragoza, España
}

\begin{abstract}
Resumen. La relevancia del sector vitivinícola en España y su conexión con el desarrollo del entorno rural, así como la preocupación de la población por los aspectos medioambientales y sociales, justifican el creciente interés por incorporar prácticas verdes y sostenibles en el turismo del vino. Además, dentro de los enoturistas existe un segmento cada vez más creciente que demanda servicios y destinos turísticos responsables con el medio ambiente y la sociedad, en general.
\end{abstract}

Teniendo en consideración esta demanda creciente, las bodegas deberían atender este segmento de consumidores que reclaman un turismo de vino sostenible. Para poder llevar a cabo una sólida iniciativa enoturista sostenible, este trabajo propone un marco teórico basado en un enfoque multinivel, que incluye además de las tradicionales perspectivas multi-stakeholders y multi-motivacional, una perspectiva multidimensional que recoge los aspectos claves del concepto de sostenibilidad, es decir, el económico, el social y el medioambiental.

\section{Introducción}

El sector del vino en España tiene una gran importancia, debido a su valor económico, social, cultural y ambiental. De hecho, según datos de la Organización Internacional de la Viña y el Vino (OIV), en 2016 la superficie de viñedos en España, con 975 mil hectáreas, era la mayor en el mundo (concretamente representaba un $14 \%$ de la superficie de viñedos mundial y un $30 \%$ de la europea). Por otra parte, España, con 39,4 millones de hectolitros, era el tercer país productor de vino en el mundo. En cuanto al consumo, en 2016, España ocupaba el séptimo lugar en el ranking mundial, con 9,9 millones de hectolitros consumidos. Otro dato relevante es que España fue el mayor exportador de vino, con 22,9 millones de hectolitros en términos de volumen y 28.900 millones de euros en términos de valor, lo que representó una cuota de mercado global del $22 \%$.

No obstante, la importancia del sector del vino no se refleja únicamente en términos de su producción, sino también en el peso que supone el enoturismo o turismo del vino como destino de calidad. El sector vitivinícola crea a su alrededor todo un ecosistema de actividades que deberían estar alineadas con la sostenibilidad ambiental y cultural, el desarrollo económico del medio rural y la desestacionalización de la demanda turística.

Los datos aportados por el noveno informe anual de la Asociación Española de Ciudades del Vino (ACEVIN) ponen de manifiesto que 2016 fue un excelente año para el enoturismo y las cifras de visitantes reflejan la consolidación del turismo del vino dentro de la oferta turística española. El creciente interés mostrado por los turistas del vino ha impulsado la proliferación de eventos de vino y el crecimiento del negocio del enoturismo.

En líneas generales, el enoturismo se relaciona con un turismo gastronómico, cultural y de naturaleza. El turismo del vino puede ser definido como todas aquellas visitas a viñedos, bodegas, festivales del vino, catas de vino y/o experiencias respecto a todos los atributos de la uva y del vino de cualquier zona vitivinícola, que el visitante tiene como primer factor motivador [1].

El turismo del vino es uno de los productos turísticos más complejos en cuanto a su estructura, gestión, promoción y comercialización. En él intervienen dos sectores de gran importancia estratégica para el territorio que tradicionalmente no mantenían ningún vínculo entre sí: el turismo y el sector vitivinícola.

La cultura del vino debe ser el eje temático del enoturismo $\mathrm{y}$, el vino, el elemento cultural que cuenta con la entidad suficiente como para apoyar y potenciar una amplia variedad de servicios, actividades, patrimonio, folclore y experiencias turísticas.

No obstante, el modelo español de enoturismo presenta aspectos mejorables, ya que, por ejemplo, se ha desarrollado sin tener en cuenta, en muchos casos, a todos sus agentes. Mantiene como producto básico la visita a la bodega bajo la denominación de experiencia enoturística, cuyo hilo conductor es el proceso de elaboración del vino. Está centrado en el corto plazo y, por ello, en demasiadas 
ocasiones, no tiene en cuenta el impacto emocional que se genera, percibiendo como un fracaso el que un turista no compre vino directamente en la bodega tras la visita. El vino es el eje vertebrador de la experiencia turística; sin embargo, para lograr la estabilidad de este producto/servicio, el enoturismo debe ser algo más que turismo enológico.

Además, su papel clave en el desarrollo local y rural hace que el interés por el tema de la sostenibilidad en el vino esté más que justificado. De hecho, en la industria del vino, las preocupaciones ambientales están dando forma a la actual demanda turística, centrada en proteger los recursos, en especial, los viñedos y paisajes, ya que la preservación del territorio es un factor altamente valorado por el enoturista experimentado. Pero dentro de los recursos turísticos medioambientales no todos son renovables, de ahí que haya que poner en marcha mecanismos para asegurar que la afluencia de visitantes no ponga en peligro dichos recursos. El desarrollo turístico se debe planificar y gestionar de forma que no cause serios problemas ambientales o socioculturales.

Así, frente a un turismo de masas, en el modelo de enoturismo sostenible debe primar una producción a pequeña escala, buscando al máximo la autenticidad en la experiencia enoturística, dirigida a un público individualizado y exigente. En este sentido, una casa rural con encanto para un destino centrado en el vino, tendría un valor cualitativo más alto que un hotel de cinco estrellas.

También se debería fomentar la implantación y el desarrollo de sistemas de gestión de la calidad medioambiental por parte de los territorios enoturísticos, concediendo prioridad al modelo de las 3R (reducir, reutilizar y reciclar). Un medio ambiente degradado resulta molesto e incluso peligroso para los residentes y puede disuadir a los visitantes. Para conseguirlo, las Administraciones deberían apoyar medidas para reducir la polución ambiental, visual y acústica (planes de embellecimiento, soterrado de cables en zonas urbanas, y regulación de los ruidos y publicidad en espacios públicos, entre otros).

Para mejorar la calidad medioambiental del enoturismo, las empresas, y no solo las administraciones, deben asumir una mayor responsabilidad, en la línea de impulsar la aplicación de medidas responsables con el medio ambiente, así como sistemas de auditoría ambiental, tanto para empresas como para destinos enoturísticos. En definitiva, se trataría de crear una cultura de la responsabilidad medioambiental integrada dentro de sus procesos de gestión.

Además, un enoturismo responsable también puede contribuir a aumentar el nivel de conocimiento y respeto por el medio natural, así como a generar recursos que puedan invertirse en su conservación. Este turismo "verde" del vino, encaja bien con visitantes de una elevada conciencia ambiental, permite crearla o aumentarla en aquellos que no la tienen y desarrolla iniciativas de turismo alineadas con los intereses de la población local.

El territorio de una ruta vitivinícola desempeña un papel muy importante, al estar compuesto por espacios naturales, urbanos y por un entorno singular que le confiere su carácter distintivo. En este contexto, el desarrollo de proyectos turísticos sostenibles favorece la conservación y mejora de los recursos naturales y culturales presentes en cada territorio, lo que mantendría intacto el potencial de generar riqueza en la zona de las próximas generaciones.

Para que el cambio de modelo sea una realidad, sería necesario que se instrumentaran acciones de formación, educación y conciencia pública del cuidado de todos los espacios dentro del propio territorio. Igualmente necesario sería poner en marcha programas de protección de recursos naturales. Es preciso resaltar también la necesidad de integrar los esfuerzos de coordinación y de apoyo de las administraciones públicas y del sector privado en el desarrollo del producto enoturístico sostenible y trabajar bajo un enfoque holístico.

Teniendo en consideración todo lo anterior, este trabajo presenta un marco teórico que permita llevar a cabo múltiples propuestas de enoturismo sostenible desde un enfoque multi-nivel, que incluya además de las tradicionales perspectivas multi-stakeholder y multimotivacional, una perspectiva multi-dimensional que contemple los tres aspectos claves del concepto de sostenibilidad (económico, social y medioambiental).

El resto del trabajo se organiza del siguiente modo. La sección 2 presenta la aproximación multinivel utilizada en el trabajo y la sección 3 incluye las conclusiones más relevantes del trabajo.

\section{Enfoque multi-nivel}

En esta sección presentamos la metodología que se va a utilizar en el trabajo que está basada en un enfoque multinivel siguiendo las ideas de [2]. En primer lugar, se lleva a cabo una perspectiva multi-stakeholder que permita determinar todos los grupos de interés o agentes involucrados en el producto/servicio del enoturismo. En segundo lugar, realizamos un análisis multi-motivacional que permita discriminar las principales razones (cultura, gastronomía o naturaleza) que guían la práctica enoturística de una persona. Finalmente, se añade una perspectiva multi-dimensional para analizar e identificar estrategias de actuación desde la óptica de la triple línea de sostenibilidad: económica, social y ambiental.

La Figura 1, mediante un cubo de Rubik, muestra gráficamente el esquema seguido en el trabajo. Cada pequeño cubo recogería un grupo concreto de actividades enoturísticas que se abordarían desde las tres perspectivas: stakeholder, motivación y dimensión.

\subsection{Nivel Multi-stakeholders}

Es evidente que una mayor participación de todos los agentes involucrados en el producto/servicio del enoturismo es crucial para introducir la sostenibilidad como alternativa al actual modelo imperante.

La teoría de los stakeholders ha tenido un desarrollo vertiginoso después de que [3] irrumpiera con esta teoría 
como enfoque y respuesta al cambio en las condiciones actuales del entorno empresarial. Entre estas condiciones se puede citar: la globalización de los mercados de capital, el aumento de los inversores institucionales, el mayor activismo por parte de los accionistas y las expectativas de los grupos de interés [4].

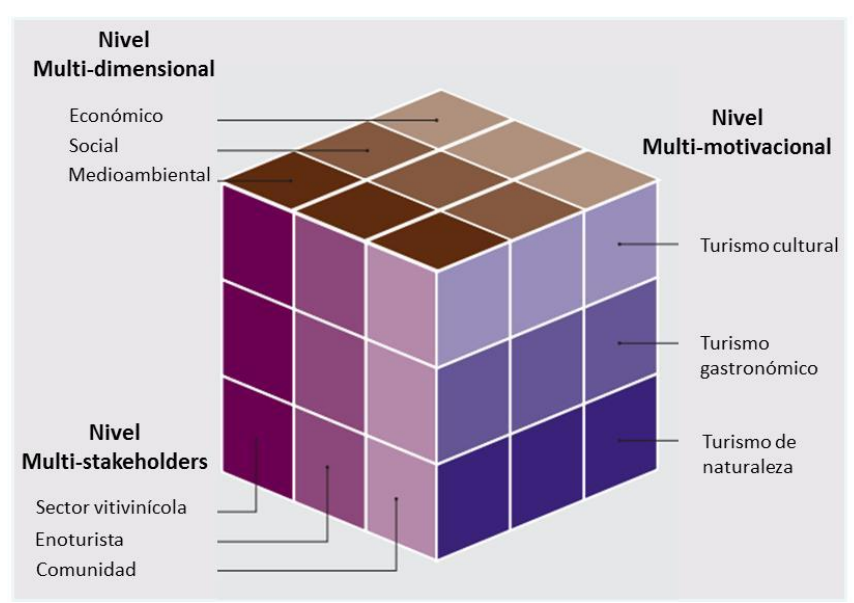

Figura 1. Esquema multi-nivel basado en [5] con las perspectivas: multi-stakeholder, multi-motivacional y multi-dimensional

Esta teoría ha sido empleada con éxito para explicar las relaciones entre los diferentes grupos de interés y la empresa en cuestiones ambientales [5-12]. En el caso del turismo, específicamente en las empresas hoteleras, se han desarrollado un amplio número de investigaciones que relacionan la gestión ambiental con los stakeholders [11, 13-15], reconociéndose como relevante la influencia de los stakeholders.

La teoría del Stakeholder [3] concibe a una empresa como un nexo de actores cuyos intereses y beneficios son mutuos. Esta teoría justifica que una empresa no solo deba satisfacer los intereses de los accionistas sino también los de todos los grupos que puedan afectar o verse afectados por las acciones de la empresa, y sin los cuales la compañía no podría existir. Muchos autores $[3,16]$ han definido y clasificado los grupos de interés en las organizaciones. En este trabajo extrapolamos esta definición de grupos de interés al ámbito que nos ocupa, el concepto de enoturismo sostenible. Por tanto, denotaremos stakeholder a cualquier persona, grupo o entidad que tenga un vínculo directo o indirecto en dicho producto/servicio. La Tabla 1 contiene una descripción detallada de los grupos de interés que tienen un interés directo o indirecto en el concepto de enoturismo sostenible.

El enoturismo se ha convertido en un producto de gran interés para una parte creciente de turistas. Pero para poder realizarlo de una forma sostenible, es imprescindible que todos los actores colaboren. El primero de ellos es la bodega - un elemento fundamental en la consolidación de este producto-. En este sentido, no hay duda de que la creación de rutas vinícolas es un buen acicate para involucrar a todos los agentes de la zona y articular un producto turístico sostenible basado en la cultura del vino.

La visita a bodegas, tradicionalmente, respondían a una labor de fidelización de clientes, que se llevaba a cabo con mucho cariño en el propio corazón del negocio pero sin considerarlo como una nueva fuente de ingresos. Cierto es que cuando se habla de enoturismo, la bodega pasa a ser un atractivo de primer orden, un elemento clave si lo que se pretende es articular una oferta turística en torno al vino. Pero, para conseguir resultados sostenibles, es requisito indispensable que la bodega tenga conciencia turística y ecológica; y, por tanto, ha de ver en el enoturismo sostenible una nueva unidad de negocio dentro de sus instalaciones. Y, por este motivo, ha de empezar a trabajar con un enfoque sostenible en la encrucijada entre los dos sectores: el enológico y el turístico. La bodega debe estructurar una nueva estrategia de negocio vinculada al turismo sostenible.

Tabla 1. Grupos de interés de un enoturismo sostenible.

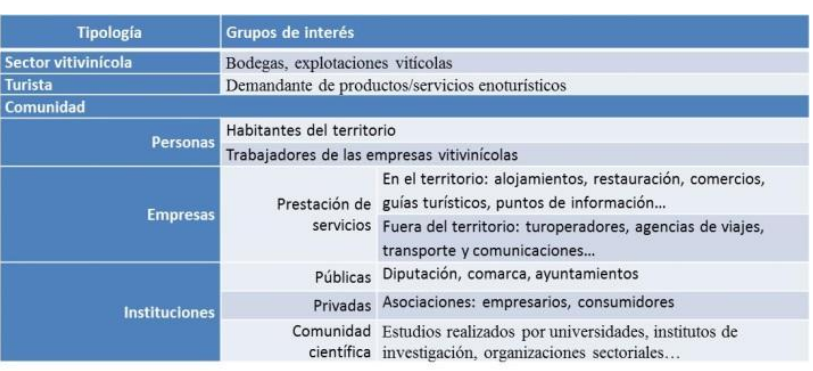

A fin de recibir visitantes, es esencial habilitar convenientemente las instalaciones de la bodega. Ésta ha de saber revalorizarlas adaptándolas a los tiempos presentes en materia de seguridad y accesibilidad; pero, sobre todo, debe encontrar elementos que vertebren una visita y promover espacios donde interpretar el proceso de elaboración del vino. En definitiva, el futuro pasa por un aprendizaje que genere experiencias vinculadas al terruño, a las instalaciones vinícolas, a las personas que intervienen en el proceso de elaboración del vino y a su consumo.

La red Great Wine Capitals realizó una encuesta, en 2009, a 454 empresas con actividades de enoturismo a nivel mundial y obtuvo que, un $83 \%$ ofertaban degustación de sus vinos, un $75 \%$ visitas guiadas, un $40 \%$ tenía una oferta gastronómica en la bodega, un 29\% incluía algún servicio de alojamiento (hotel, casa rural etc.), un 20\% algún tipo de actividades deportivas en sus instalaciones, un $20 \%$ actividades culturales, un 17\% albergaban en sus bodegas algún tipo de exposición de carácter temporal; un 10\% ofrecía en sus instalaciones un espacio museístico de manera permanente y un $4 \%$ realizaban terapias basadas en los beneficios del vino y la viticultura. Sin embargo, esta encuesta no nos dice cómo se llevan a cabo esas actividades, es decir, si se hacen o no de una manera sostenible tanto desde el punto de vista económico, social y medioambiental.

Otro de los agentes involucrados en el producto es el turista. El consumidor está cambiando y, cómo no, también sus hábitos vacacionales. Actualmente realiza salidas con mayor asiduidad que antes, si bien más fragmentadas y cortas. Este consumidor elige lugares más próximos para sus escapadas, valora los destinos con conciencia ambiental vinculados a la naturaleza y a un estilo de vida saludable. Trekkings, rutas en bicicleta y actividades deportivas son valores en alza. El turista, en su tiempo de ocio, busca los alicientes del llamado Life Style: 
gastronomía, actividades recreativas, balnearios, "shopping", etc. Normalmente, los destinos escogidos van asociados a algún atributo emocional e intangible, $y$ se busca lo auténtico, lo singular. En definitiva, el turista tiene sed de novedades; quiere vivir experiencias en sus viajes y anhela nuevos productos turísticos que lo satisfagan y cumplan con sus expectativas iniciales.

El enoturismo es un turismo de proximidad básicamente, pues el $40 \%$ de los turistas del vino, lo son del mismo país que visitan. Del conjunto total, el $25 \%$ de visitantes son locales. Por término medio el 35\% de los enoturistas son extranjeros. Los países que más enoturismo exportan son Holanda y Reino Unido, seguido por Estados Unidos y Canadá. Dos tercios de enoturistas son hombres y el tercio restante pertenece al género femenino. El visitante medio $(45 \%)$ tiene una edad entre 36 y 55 años. Los maduros de más de 56 años también son grandes aficionados al enoturismo (30\%). El enoturismo es una actividad fuertemente vinculada a las vacaciones pues el $70 \%$ de enoturistas llegan a los territorios de vino durante el verano. El otoño es la otra temporada importante para los enoturistas, concentrando el 15,3\% de las visitas totales.

Se necesitan pueblos impecables en cuanto a servicios, señalización, gasolineras, hoteles, restaurantes, tiendas especializadas, etc. Se deben diseñar servicios de enoturismo para un día, 2, 3 o 5 días, por ejemplo.

Para los empresarios turísticos, de ocio y oferta relacionada, es una importante oportunidad para generar demanda adicional a su producto turístico tradicional, el turismo rural que, a pesar de su importante crecimiento en los últimos años, sufre de la problemática de una excesiva estacionalidad en fines de semana, puentes y época estival, así como de una cada vez más feroz competencia entre destinos y establecimientos por captar flujos de demanda.

Finalmente, para los territorios vitivinícolas constituye una oportunidad única para crear un producto turístico singular, que corresponde a las nuevas tendencias de la demanda y que, mediante una planificación adecuada, puede convertirse en un "motor" para el desarrollo turístico sostenible de un territorio, generalmente caracterizado por una excesiva dependencia del sector vitivinícola.

Un enoturismo sostenible requiere trabajar en estrecha colaboración entre la administración pública (turística, medio rural, local, provincial y regional) y el sector privado (turístico y vitivinícola).

Resumiendo, el beneficio derivado de un producto de enoturismo sostenible debe ser múltiple. Para el turista, al asegurar que el diseño del producto turístico y la prestación de los servicios que implica, responderá satisfactoriamente a sus necesidades. Para la administración local que podrá aprovecharse de los múltiples beneficios: p.ej. intercambio de opiniones con otras administraciones locales, economías de escala en la realización de proyectos $y$ en la promoción $y$ comercialización, etc. Para el empresario que obtendrá una guía para definir o adaptar sus instalaciones y servicios a los requerimientos de competitividad establecidos por los clientes y el entorno y para el residente puesto que el desarrollo y gestión del destino enoturístico tendrá en cuenta su punto de vista sobre el tema, una necesaria combinación entre crecimiento $\mathrm{y}$ conservacionismo.

\subsection{Nivel Multi-motivacional}

Existen numerosas evidencias sobre la gran importancia que poseen las motivaciones en el comportamiento y proceso de elección del turista. Por lo tanto, parece necesario comprender las motivaciones que influyen en la elección del producto enoturístico. La motivación permite dar respuesta a tres cuestiones esenciales: las razones para viajar o por qué; la elección específica o dónde; y los beneficios buscados en el destino o satisfacción [17-18]. Para [19], la motivación es una variable muy importante para explicar la conducta turística.

Asimismo, la motivación se ha estudiado conjuntamente con la segmentación del mercado [20-25], así como con la lealtad con el producto o destino.

En términos generales, los turistas viajan bien porque son empujados por motivos o variables internas o bien porque son atraídos por fuerzas externas del destino. Los factores de empuje (push factors) están relacionados con aspectos internos y emocionales, como el deseo de escapar, de descansar y relajarse, de aventura o de interacción social. Los factores de atracción (pull factors) están conectados con aspectos externos, cognitivos y de situación, como los atractivos del lugar, las infraestructuras recreativas o los escenarios culturales y naturales [26-28]. Una cuestión de interés es que la bodega puede trabajar en ambos tipos de estrategias simultáneamente, una más a nivel sectorial basada en factores push, para crear una intrínseca conciencia de cultura del vino a través de sensaciones y otra más basada en factores pull ligada al territorio, a través de una buena oferta.

Dentro de los factores pull, según un estudio realizado por la Organización Mundial del Turismo (OMT), la oferta cultural, la naturaleza y la gastronomía, son, por ese orden, los motivos principales que llevan a los enoturistas a visitar un destino.

Recorrer un paisaje vitivinícola, visitar bodegas, aprendiendo su cultura mientras se catan vinos y se prueban los alimentos típicos de la zona, pudiendo incluir actividades terapéuticas, deportivas o de relax, se ha convertido en una forma de viajar con una demanda cada vez más en alza.

La autenticidad de la vivencia enoturística debe preservarse. El folclore, la historia y las tradiciones redondean esa vivencia cultural del enoturista, por tanto, preservar y potenciar el patrimonio del destino es decisivo.

En cuanto a la motivación cultural, el interés por los destinos rurales en España ha aumentado notablemente a lo largo de los últimos años. Estos lugares invitan a descubrir pintorescos pueblos, villas, castillos y pequeñas ciudades medievales, ofreciendo también la posibilidad de hospedaje en pequeñas casas rurales y magníficos hoteles de interior. 
Por otra parte, la gastronomía local también forma parte del patrimonio de un destino enoturístico y merece una especial atención dada su complementariedad con el elemento vino. Por tanto, conocer los hábitos y preferencias de un enoturista es importante para establecer la oferta gastronómica, el tipo de alojamientos, el tipo de establecimientos en los que les gusta comer o qué actividades gastronómicas buscan para complementar su viaje.

Según el segundo estudio de la demanda de turismo gastronómico, un $76,2 \%$ de los españoles ha realizado algún viaje o escapada con la intención de disfrutar de la gastronomía en los últimos dos años. El 62,4\% de estos turistas afirma que la gastronomía influye en gran medida en la elección del destino. El 42,6\% de los desplazamientos que se realizan a destinos nacionales cercanos (menos de $200 \mathrm{~km}$ ) con la intención de disfrutar de la gastronomía son viajes en el día sin pernoctación en el destino, en los que el visitante se desplaza para disfrutar de una comida en un restaurante, ir de tapas o visitar una bodega. El sector de la restauración y la hostelería se convierte, por tanto, en el escaparate gastronómico del territorio, por ser éste el principal espacio en el que entran en contacto el visitante y el patrimonio gastronómico de un lugar. Los turistas gastronómicos también aprovechan estos viajes para comprar productos locales $(62,9 \%)$, visitar mercados gastronómicos $(59,0 \%)$ y realizar actividades enoturísticas, tales como visitar bodegas $(49,1 \%)$ y comprar vinos $(41,5 \%)$.

Por último, el entorno natural es otro aspecto que no hay que olvidar puesto que admirar la flora, la fauna, bellos parajes naturales, hermosos lagos y montañas, preciosos ríos y acantilados es un interesante reclamo para un número creciente de enoturistas. Entre las personas que practican el turismo de naturaleza se pueden encontrar perfiles muy variados. Así, este tipo de turismo lo practican los naturalistas que se documentan sobre los lugares que van a visitar; los individuos que quieren sentir la tranquilidad y las oportunidades de ocio que la naturaleza les ofrece; los estudiosos, que buscan el conocimiento y la percepción de los espacios naturales o las personas mayores que buscan disfrutar del paisaje y de su belleza natural, entre otros.

Según [29], conocer la conducta del turista permite entender mejor las razones por las cuales se realiza la visita, las necesidades que tienen los visitantes y el uso que se le va a dar al lugar.

\subsection{Nivel Multi-dimensional}

El enoturismo posee un impacto que puede ser positivo y negativo, como resultado de la interacción con los diferentes factores del entorno que lo rodea. Este impacto puede deberse a los derivados de la fase de construcción e implantación del equipamiento turístico y las infraestructuras, a los producidos en su estado de funcionamiento operativo y a los generados por las actividades que realizan los propios turistas. Para llevar a cabo un análisis de sostenibilidad del enoturismo, deben analizarse los impactos económicos, sociales y ambientales. Es importante precisar que los impactos no son solamente perjudiciales o dañinos, al contrario, existen muchos beneficios como resultado de la actividad enoturística que pueden ser utilizados para revertir el impacto negativo de la misma.

En general, la mayoría de las consecuencias económicas de la realización de actividades enoturísticas podrían ser positivas, por lo menos a corto plazo. Tanto los gastos de los turistas que visitan una ruta vitivinícola como la inversión realizada por los dueños de las bodegas conllevan un conjunto de beneficios para muchas actividades económicas locales (hoteles, restaurantes, taxis, autobuses, etc.). Debido a que el turismo es una industria que depende en gran medida del factor humano, favorece la creación de empleo tanto en el sector turístico como en otros sectores impulsados por el turismo. La actividad enoturística no solo elevaría la renta en el área donde se desarrolla, sino también mejoraría su distribución, tanto en términos poblacionales como en términos de contribución al equilibrio regional. En general, se puede afirmar que el enoturismo representa una posibilidad de mejora económica en el nivel de vida de la población residente.

No obstante, todos los impactos en el aspecto económico no son positivos. Uno de los problemas derivados de la actividad turística son las fluctuaciones de su demanda, esta demanda es estacional y sensible a variaciones en el precio y a las modas, lo cual puede traer consigo inestabilidad en las zonas donde se desarrolle.

El impacto social de la actividad enoturística es evidente también desde sus propios inicios. El enoturismo puede traer consigo ventajas para las localidades de la zona, como es el caso de preservación y rehabilitación de monumentos, edificios, lugares históricos y creación de museos, centros de interpretación y monumentos de interés cultural, etc. Otra de las ventajas más comunes para la región donde se desarrolla la actividad turística puede ser la mejora de las infraestructuras, instalaciones y servicios. Por ejemplo, la atención sanitaria, alumbrado, recogidas de basuras, mejora de las comunicaciones, nuevas sucursales de entidades financieras. Así pues, la calidad de vida de los residentes aumenta.

Sin embargo, no todos los impactos sociales son positivos ya que el desarrollo de la actividad enoturística puede provocar a veces una pérdida de la identidad cultural y de tradiciones, así como una estandarización y banalización de la cultura. Además, la proliferación de visitantes puede traer consigo que los habitantes de la zona encuentren dificultades para mantener áreas familiares, con cierta intimidad y libres de turistas.

El impacto ambiental es quizás el efecto más visible, partiendo de la etapa de construcción de las instalaciones hasta la propia actividad del turista. Por ello, la primera de las fases, construcción e instalación, es la etapa que mayor planificación debería tener, teniendo en cuenta que en ese momento se deciden muchos de los elementos que afectarán al entorno tanto a corto como a largo plazo. Durante la implantación de las instalaciones se consumen muchos materiales y recursos, partiendo desde el uso del suelo donde se construye la instalación hasta el consumo de materiales de construcción y energía. Este consumo es relevante teniendo en cuenta la irreversibilidad del proceso, principalmente tratándose del deterioro del suelo. 
Sin embargo, el mayor impacto negativo puede ser en el aspecto visual y arquitectónico. La mediocridad en un diseño y la ubicación de las construcciones configuran una total contaminación arquitectónica visual destructora del paisaje.

Otro elemento de gran impacto medioambiental es la generación de residuos, tanto durante la construcción y puesta en marcha, como principalmente en la etapa de explotación del turismo. En esta última etapa se generan residuos sólidos, en su mayoría residuos domésticos, con una gran diversidad, desde botellas de cristal, latas, papel hasta residuos alimenticios, haciendo de esta forma muy difícil su clasificación y tratamiento.

Otro recurso que es de vital importancia y que es uno de los más afectados por la actividad turística es el agua, en sus diversas formas: su uso indiscriminado, específicamente del agua potable, en zonas donde por lo general este recurso escasea; y la contaminación como resultado de aguas residuales. Esta polución del agua trae consigo la pérdida de la biodiversidad, el incremento de la turbiedad del agua y peligros sanitarios, entre otros.

Asimismo, el ruido y la contaminación del aire están asociados a la explotación y a la actividad del turista.

Sin embargo, cuando la actividad enoturística se realiza de forma consciente y planificada puede redundar en mejoras para la zona donde se concentra. Esta tendencia a proteger valores ambientales que de otra forma no tuvieran ningún valor económico es lo que puede hacer del enoturismo uno de los sectores fundamentales para apostar por la sostenibilidad.

\section{Conclusiones}

El perfil del turista es cambiante, el turismo que se practica a día de hoy no tiene nada que ver con el de hace unos años. Como dice el sociólogo Gilles Lipovetsky "el ocio será cada vez más híbrido, se entrelazará con la cultura, el arte, la estética, la gastronomía..."

El turismo en general, y el enoturismo, en particular, está considerado como un motor no desdeñable de la actividad empresarial, por tanto, un impulso positivo en el sector enoturístico redundaría en la mejora y crecimiento del resto de actividades económicas relacionadas con el mismo. Todos queremos que el enoturismo crezca, nos encantaría ser líderes mundiales, que nuestra zona fuese conocida en el mundo entero por lo que ofrecemos. Pero, desde un punto de vista motivacional, ¿se busca volumen o calidad de turismo?, ¿turismo low cost o turismo premium?, ¿se debe sectorizar orientándolo a segmentos como deportistas, turismo familiar, senior, joven, etc.?, ¿sabemos que oferta cultural, natural y gastronómica quiere cada uno de ellos?. Por tanto, para un buen diseño del producto, parece lógico comenzar definiendo el público objetivo, y en base a él actuar: conociendo a quién se dirige se personalizaran las experiencias y se conseguirán clientes satisfechos. Desde un punto de vista multi-stakeholders, ¿estamos preparados?, ¿sabríamos gestionar un crecimiento de manera correcta para satisfacer las necesidades del turista, las del empresario, las de la población local y las del medio ambiente?. Para un enfoque multi-nivel faltaría incorporar a estas preguntas el concepto de sostenibilidad en sus tres dimensiones, la social, la económica y la medioambiental, que creemos contienen la respuesta de futuro a las cuestiones planteadas.

El enoturismo ha ido adoptando iniciativas de protección medioambiental, aunque todavía no sean suficientes para salvaguardar la naturaleza; los visitantes acuden cada vez con mayor frecuencia a destinos y lugares naturales que deben ser debidamente protegidos para que las personas puedan disfrutarlos en el futuro. La gastronomía y la cultura también se pueden relacionar con el enoturismo sostenible, potenciando el consumo de productos de la zona, utilizando proveedores locales, revitalizando las costumbres y tradiciones: artesanía, festivales, folklore, etc., lo que generará un intercambio cultural entre visitantes y residentes.

Este enfoque multi-nivel trata de buscar soluciones a la fase de saturación dentro del ciclo evolutivo de cualquier actividad turística. No hay que olvidar que, mientras que en las primeras fases de aparición de cualquier modalidad turística se genera un elevado entusiasmo por parte de la población residente, al percibirlo como una buena opción de desarrollo a la que hay que sacar partido; a medida que se alcanzan niveles de saturación, los residentes necesitan facilidades adicionales para poder aceptarla. Posteriormente, dicha actividad pasa a ser considerada como la causante de todos los males del destino, para los residentes todas estas injerencias en la cultura local provocan un sentimiento de pérdida de lo que les pertenece y trae consigo elementos hostiles que atentan contra el ambiente acogedor y hospitalario que requiere la industria turística. De manera que en la etapa final, el destino ha perdido todos los atractivos que originalmente atrajeron a los turistas.

Por tanto, circunscribiéndolo al caso del enoturismo, el enoturismo sostenible puede ser la solución a estos inconvenientes. Un turismo más respetuoso con el patrimonio natural y cultural, así como con la población local, menos intensivo en el número de turistas y que se ajuste mejor a la capacidad de ocupación del destino, más controlado en los impactos negativos causados por los turistas y que no comprometa la capacidad de crecimiento y desarrollo de las generaciones futuras.

\section{Agradecimientos}

Este trabajo ha sido financiado por los proyectos ECO2016-77-P y ECO2016-79392-P (AEI/FEDER, UE).

\section{Referencias}

1. C.M. Hall. Wine tourism in New Zealand In G. Kearsley (ed) Tourism Down Under II, Towards a More Sustainable Tourism Conference Proceedings. 99, 109-119. Centre for Tourism, University of Otago (1996)

2. S. Qayum, S. Gold, Y. Glemarec Multi-Stakeholder 
Decision-Making A Guidebook for Establishing a Multi-Stakeholder Decision-Making Process to Support Green, Low-Emission and Climate-Resilient Development Strategies, United Nations Development Programme, UNDP (2012)

3. R. E. Freeman. Strategic Management: A Stakeholder approach, Boston: Pitman (1984)

4. T.I. Nwanji, K.E. Howell. Shareholdership, stakeholdership and the modern global business environment: A survey of the literature. Journal of interdisciplinary economics. 18, 4, 347-361 (2007).

5. J. K. Thompson, S. L. Wartick, H.L. Smith, J. E. Post. Integrating Corporate Social Performance and Stakeholder Management: Implications for a Research Agenda in Small Business. IN PRESTON, L. E. (Ed.) Research in corporate social performance and policy. . A Research Annual Greenwich, Conn. and London, JAI Press (1991)

6. R. E. Freeman. The politics of stakeholder theory: Some future directions. Business Ethics Quarterly, 4, 4, 409-421 (1994)

7. P. Shrivastava. The role of corporations in achieving ecological sustainability. Academy of Management Review, 20, 4, 936-960 (1995)

8. S. Fineman, K. Clarke. Green stakeholders: Industry interpretations and response. Journal of Management studies, 33, 6, 715-730 (1996)

9. G. Azzone, M. Brophy, G. Noci, R. Welford W. Young. A stakeholders' view of environmental reporting. Long range planning, 30, 5, 699-709 (1997)

10. I. Henriques, P. Sadorsky. The relationship between environmental commitment and managerial perceptions of stakeholder importance. Academy of management Journal, 42, 1, 87-99 (1999)

11. M. J. Alvarez Gil, J. Burgos Jiménez, J.J. Céspedes Lorente. An analysis of environmental management, organizational context and performance of Spanish hotels, Omega, 29, 6, 457-471 (2001)

12. B.R. Agle, T. Donaldson, R.E. Freeman, M.C. Jensen, R. K. Mitchel, D. J. Wood. Dialogue: Toward superior stakeholder theory. Business Ethics Quarterly, 18, 2, 153-190 (2008)

13. J. Céspedes-Lorente, J. Burgos-Jiménez, M. J. Álvarez-Gil. Stakeholders' environmental influence. An empirical analysis in the Spanish hotel industry. Scandinavian Journal of Management, 19, 3, 333358 (2003)

14. J. Rivera. Institutional Pressures and Voluntary Environmental Behavior in Developing Countries: Evidence From the Costa Rican Hotel Industry. Society \& Natural Resources, 17, 1, 779-797 (2004)

15. A. Kasim. Towards a Wider Adoption of
Environmental Responsibility in the Hotel Sector', International Journal of Hospitality \& Tourism Administration, 8, 2, 25-49 (2007)

16.

A. Campbell. Stakeholders: The case in favour. Long Range Planning 30, 446-49 (1997)

17. S. Baloglu, K. W. McCleary. US international pleasure travelers' images of four Mediterranean destinations: A comparison of visitors and nonvisitors. Journal of Travel Research, 38, 2, 144-152 (1999)

18. M. Castaño, A. Moreno, S. García, A. Crego. Aproximación psicosocial a la motivación turística. Estudios turísticos, 158, 5-42 (2003)

19. D.A. Baker, J. L. Crompton. Quality, satisfaction and behavioral intentions. Annals of Tourism Research, 27, 3, 785-804 (2000)

20. M. Cervantes, A. González, N. Muñiz. La incidencia de los estilos de vida en la segmentación del mercado turístico: aplicación a la población urbana de Castilla y León. Revista de Investigación Económica y Social de Castilla y León, 2, 41-56 (1999)

21. C. K. Lee, Y. K. Lee, B. E. Wicks. Segmentation of festival motivation by nationality and satisfaction. Tourism management, 25, 1, 61-70 (2004)

22. A. Beh, B. L. Bruyere. Segmentation by visitor motivation in three Kenyan national reserves. Tourism management, 28, 6, 1464-1471 (2007)

23. C. H. Hsu, L. A. Cai, K. K. Wong. A model of senior tourism motivations-Anecdotes from Beijing and Shanghai. Tourism Management, 28, 5, 1262-1273 (2007)

24. D. Severt, Y. Wang, P. J. Chen, D. Breiter. Examining the motivation, perceived performance, and behavioral intentions of convention attendees: Evidence from a regional conference. Tourism Management, 28, 2, 399-408 (2007)

25. M. Devesa, M. Laguna, A. Palacios. The role of motivation in visitor satisfaction: Empirical evidence in rural tourism. Tourism Management, 31, 4, 547-552 (2010)

26. G. M. Dann. Anomie ego-enhancement and tourism. Annals of Tourism Research, 4, 4, 184-194 (1977)

27. J. L. Crompton. Motivations for pleasure vacation. Annals of tourism research, 6, 4, 408-424 (1979)

28. G. M. Dann. Tourism Motivations: An appraisal. Annals of Tourism Research, 8, 2, 189-219 (1981)

29. M. Osorio, S. Franco, I.L. Ramírez, G. Nava, G. Novo, H. Hugo. El visitante del Parque Nacional Nevado de Toluca, México. Análisis del comportamiento en un área natural protegida. Investigaciones Geográficas, 76, 55-70 (2011) 